Results reported $62 \%$ victims, $11 \%$ bullies and $27 \%$ expectators. $8 \%$ from Teachers demonstrated confusion to identify bullying and the one's involved on the situation. Bullying happens in private and public schools in similar intensity. It's more reported in children, than adolescents and the verbal teasing is the frequented form of victimization, followed by physical aggressions. According to gender, girls also reported bullying, but it's in a hidden form. 4 months post-treatment, changes were seen in resilience acquisition, bullying reduction and differences based on gender.

Bullying is a pervasive, serious problem. These research findings must be disseminated to practitioners, schools and students to help victims coping with bullying. For those who are not naturally resilient, it's necessary to teach competencies associated with resilience.

\section{AGGRESSIVE AND AND SELF-HARM BEHAVIORS IN ADOLESCENTS: THE ROLE OF EMOTION REGULATION}

doi:10.1136/archdischild-2012-302724.0443

'P Velotti, ${ }^{2} \mathrm{R}$ Castellano, 'D Cavanna. 'Department of Educational Sciences, University of Genoa, Genoa; Institute of Psychoanalytic Self Psychology and Relational Psychoanalysis, Roma, Italy

Introduction The ability to regulate emotions has been identified as a protective factor associated with a reduced risk behaviors (Schneider and Caffray, 2000). Studies show that adolescents experience more frequent and intense emotions than individuals younger or older, and that at this stage of the life cycle greatly increases the incidence of conduct disorders. However, most research on emotion regulation has focused on children, while less attention was paid to early adolescence (Eisenberg \& Morris, 2002). A better understanding of the emotional development would allow a better understanding of individual differences in regulation and behavior in this period of greatest risk.

Method The sample consisted of 740 adolescents aged between 13 and 19 years (mean age $=16.70, \mathrm{SD}=0.91$ ). For participants, attending secondary schools, are administered the following instruments: a) Difficulties in Emotion Regulation Scale (DERS: Giromini, Velotti, et al. 2012), a tool that examines the overall difficulty in regulating emotions through the evaluation of six factors; Aggression Questionnaire (AQ: Fossati et al., 2009) an instrument that measures hostility and aggression through five scales and RiskTaking and Self-Harm Inventory for Adolescents (RTSHIA: Vrouva, Fonagy, Fearon, Roussow, 2010), a self-report measure designed to assess adolescent RT and SH in community and clinical settings.

Results Preliminary results show the presence of correlations between specific difficulties in emotion regulation, hostility, risk taking and self-harm behavoir, allowing a discussion about the role of emotional regulation in this particular phase of the life cycle.

\section{SEXTING, FRAPING AND HYPER-TWEETING - WHAT SHOULD PAEDIATRICIANS BE DOING TO HELP?}

doi:10.1136/archdischild-2012-302724.0444

'NN Onugha, ${ }^{2} \mathrm{~F}$ Finlay. ${ }^{1}$ Child Health Department; ${ }^{2}$ Community Child Health, Royal United Hospital, Bath, UK

Unwanted sexual solicitation, online harassment, cyber-bullying and depression are all associated risks of social media to adolescents. More than a fifth of teenagers log on to a social media site at least ten times a day, $75 \%$ own mobile phones and of these, $25 \%$ use them for social media, $54 \%$ for texting and $24 \%$ for instant messaging. Adolescents often lack awareness of 'the digital footprint'; posting inappropriate messages, private and/or explicit pictures and videos and failing to recognise that 'what goes online, stays online'. Clinicians often see the consequences of these actions, for example, sexual abuse following meeting "friends" online, self harm following cyber-bullying and near-miss suicides following activities like "the choking game" viewed online.
Yet not all social media is bad. Professionals are in a key position to sign-post useful information and health advice via social media; promoting a healthy lifestyle, a balanced diet and sexual health screening.

The American Academy of Pediatrics (AAP) has published policy statements highlighting the impact of mass media and calling for media education to be a key goal for paediatricians. Professionals can provide anticipatory guidance to families, promoting wisemedia choices as well as discussing the potential hazards. The AAP have developed a 'Media History form' to facilitate discussion.

We believe that professionals need to work together to ensure that adolescents can enjoy the benefits offered by social media while minimising the risks - how can we best achieve this?

\section{THE RANGING NEW FEATURES AS A PRACTICAL APPROACH TO CONSTRUCT NEW PROBIOTIC CONSORTIUM FORMULAS}

doi:10.1136/archdischild-2012-302724.0445

V Lakhtin, M Lakhtin, Y Belikova, Y Agapova, Y Kulakova, S Afanasiev, V Aleshkin. G.N. Gabrichevsky Research Institute for Epidemiology \& Microbiology, Moscow, Russia

Background Probiotic human bacterial mixtures constructed are important for health [1]. The aim was to develop new perspective variants of the known Russian mixed probiotic "Acilact" (Lactobacillus strains $\mathrm{NK} 1+\mathrm{K}_{3} \mathrm{III}_{24}+100_{\text {ash }}$; all strains of human origin) using approach of ranging new parameters of cultural fluids, their fractions and components.

Methods The system of at least 32 parameters was ordered as decreased/increased sequences of 4 members (Acilact and its strains) depending on any selected parameter. The cases of leadership (inverted or not in the sequence) of Acilact or strain(s) are considered as technological advantages of consortium or strain(s).

Results and discussion Among high molecular mass components (at least $30 \mathrm{kD}$ ), protein fractions, lectin systems, protease system, oxidase-reductase system, biosurfactants, intrinsic fluorofores containing protein and non-protein components, and activities against eukaryotic human pathogens were evaluated as perspective new features and criteria for construction of new system probiotics of differentially directed action. Non-linear proportional contributions of ingredient strains into final properties of Acilact were observed. Strains as dominant leaders in some features revealed in Acilact can be detected. Examples of significantly new advantages of ingredient strain(s) or Acilact are presented. The net of ordered ranges allows additional control of the results.

Conclusion The approach developed can help in constructing qualitatively new effective and directed mixed extended probiotics based on traditionaliy used probiotic bacterial industrial strains.

Reference [1]. V.V. Pospelova. Thesis (MD) [Microbial biological preparations for correction of intestinal bacteriocenoses, their designing and using (in Russian)]. Moscow. 1979.

\section{BRONCHIAL ASTHMA AMONG UNIVERSITY STUDENTS, AJMAN, UAE}

doi:10.1136/archdischild-2012-302724.0446

'LJ John, 'M Arifulla, ${ }^{2} \mathrm{~J}$ Sreedharan. 'Pharmacology; ${ }^{2}$ Research Division, Gulf Medical University, Ajman, United Arab Emirates

Background and Aims Prevalence of wheezing and allergies among the Arab countries are on a rise due to urbanization resulting in substantial environmental changes. Prevalence of wheezing is about $14 \%$ among school children but data on adolescents in the Gulf region is lacking. This study aimed to assess the prevalence of wheezing and bronchial asthma among undergraduate students of Gulf Medical University. 